\title{
Antimuscarinic Discontinuation in Patients with Overactive Bladder in Nursing Homes: A Retrospective Study of Medicare Beneficiaries
}

\author{
Rajender R. Aparasu · Sneha Sura · Jagadeswara R. Earla · \\ Aki Shiozawa · Daniel B. Ng · Carol R. Schermer
}

Received: April 22, 2020 / Published online: July 7, 2020

(C) The Author(s) 2020

\begin{abstract}
Introduction: Although antimuscarinics form the first-line therapy in overactive bladder $(\mathrm{OAB})$, little is known regarding antimuscarinic discontinuation among $\mathrm{OAB}$ patients in nursing homes. This study examined treatment patterns and predictors of antimuscarinic discontinuation among long-term nursing home (LTNH) residents with $\mathrm{OAB}$.

Methods: The study cohort included LTNH residents (defined as residents staying $\geq 101$ consecutive days) from the Minimum Data Set linked 2013-2015 Medicare claims data. Patients with OAB were defined by OAB-related claims and medication codes. Treatment patterns and discontinuation (medication gap $\geq 30$ days) were characterized by
\end{abstract}

Digital Features To view digital features for this article go to https://doi.org/10.6084/m9.figshare.12472109.

Electronic Supplementary Material The online version of this article (https://doi.org/10.1007/s12325$020-01412-z)$ contains supplementary material, which is available to authorized users.

R. R. Aparasu $(\varangle) \cdot$ S. Sura $\cdot$ J. R. Earla Department of Pharmaceutical Health Outcomes and Policy, College of Pharmacy, University of Houston, Houston, USA

e-mail: rraparasu@uh.edu

A. Shiozawa · D. B. Ng · C. R. Schermer Astellas Pharma Global Development, Inc., Northbrook, IL, USA examining OAB-specific antimuscarinics prescribed during LTNH stays. The Andersen Behavioral Model was used to identify predisposing, enabling and need factors that predict discontinuation. Kaplan-Meier curves and multivariable Cox proportional hazards regression model were used to assess the unadjusted and adjusted times to discontinuation, respectively, among different antimuscarinics.

Results: The mean age of the study cohort $(n=11,012)$ was 81.6 years $( \pm 8.5), 74.6 \%$ were female, and $89.8 \%$ were non-Hispanic White. The mean duration of nursing home stay was 530.1 ( \pm 268.4$)$ days. The most commonly prescribed OAB-specific antimuscarinic was oxybutynin (69.8\%). Overall, $66.5 \%$ of the study cohort discontinued the index antimuscarinic. Multivariable Cox $\mathrm{PH}$ regression analysis revealed that compared to LTNH residents who initiated treatment with oxybutynin, treatment discontinuation was lower with solifenacin or fesoterodin and discontinuation was more frequent when treatment was initiated with tolterodine, darifenacin or trospium compared with oxybutynin. In addition, several need factors (comorbidities, medication use and anticholinergic burden, etc.) were associated with antimuscarinic discontinuation.

Conclusion: About two-thirds of LTNH residents with $\mathrm{OAB}$ discontinued their index antimuscarinic during their nursing home stay. There was significant variation in discontinuation based on the index antimuscarinic agent 
with lowest risk of discontiuation with solifenacin and fesoterodin. Concerted efforts to optimize antimuscarinic use are needed to improve the management of $\mathrm{OAB}$ in nursing homes.

Keywords: Adherence; Antimuscarinic; Overactive bladder; Nursing home; Urology

\section{Key Summary Points}

Why carry out this study?

Antimuscarinics are the front-line pharmacotherapy for overactive bladder $(\mathrm{OAB})$, but there is a paucity of data on treatment persistence in this setting.

The objective of the present study was to examine treatment patterns and to assess the medication discontinuation among different antimuscarinics in long-term nursing home (LTNH) residents with $\mathrm{OAB}$.

\section{What was learned from the study?}

A low proportion of LTNH residents with $\mathrm{OAB}$ under the fee-for-service Medicare plan is prescribed antimuscarinics.

Antimuscarinic discontinuation during nursing home stay was high among LTNH residents with $\mathrm{OAB}$.

Discontinuation varied based on the index antimuscarinic agent, with the lowest risk of discontinuation among LTNH residents who were prescribed solifenacin and fesoterodin.

\section{INTRODUCTION}

In 2014, there were an estimated 1.4 million nursing homes residents in the USA [1]. Although nursing home residents may vary in terms of their physical and mental ability and capacity for self-care, residents on average have a higher disease burden compared with community-dwelling individuals [2]. Urinary incontinence (UI) is a symptom accompanying many conditions common among older adults, affecting as many as $50-70 \%$ of nursing home residents [3, 4]. Many cases of UI are associated with overactive bladder (OAB), a clinically defined symptom complex characterized by urgency with or without incontinence, often accompanied by frequency and nocturia $[5,6]$. In a large claims-based study, nursing home residents with $\mathrm{OAB}$ and/or UI had a higher prevalence of depression $(47.7 \%$ vs. $32.4 \%)$, cerebrovascular accidents $(20.4 \%$ vs. $10.4 \%)$ and dementia (40.8\% vs. $35.4 \%)$ compared with those without $\mathrm{OAB}$ and/or UI [7]. In addition to the clinical burden associated with $\mathrm{OAB}$ among nursing home residents, the economic burden is substantial. Although recent data are lacking, in 2000 , the total direct cost of $\mathrm{OAB}$ in nursing homes, including diagnostic, treatment, routine care and health-related consequences (i.e., falls, skin conditions and urinary tract infections), was estimated at 3.5 billion (year 2000 USD) [8].

Antimuscarinics such as oxybutynin, tolterodine, solifenacin, darifenacin, fesoterodine and trospium are approved as a first-line pharmacotherapy for OAB in the US, and, when used appropriately, are an effective and well-tolerated treatment that improves quality of life in patients with OAB [9]. Although there are limited data on pharmacotherapy treatments for $\mathrm{OAB}$ in nursing homes, antimuscarinics appear to be widely used [7]. In a retrospective analysis of nursing home residents, almost $80 \%$ of those with $\mathrm{OAB}$ were treated with an antimuscarinic (primarily oxybutynin extended-release) [7]. Despite their effectiveness at managing $\mathrm{OAB}$ symptoms, adherence (often defined as patients who take at least $80 \%$ of their prescribed medication or a medication possession ratio [MPR] $\geq 0.8$ ) [10] to antimuscarinics is low [11, 12]. Among community-dwelling $\mathrm{OAB}$ patients, estimates of antimuscarinic adherence range from 14 to $35 \%[11,12]$. In a national survey, dissatisfaction with treatment effectiveness and side effects, which can include dry mouth, blurred vision, headache and constipation, have been cited as reasons for $\mathrm{OAB}$ treatment discontinuation [13]. Based on a systematic review, the treatment discontinuation rate among $\mathrm{OAB}$ patients was reported in the range of $43-83 \%$ [14]. 
To date, most studies on antimuscarinics have focused on adherence and have been conducted among community-dwelling individuals $[11,12,15]$; there is thus a paucity of data regarding antimuscarinic discontinuation among nursing home residents with OAB. Only one study examined discontinuation in US outpatient settings and found that $72 \%$ discontinued their antimuscarinics, suggesting a significant need to improve adherence [16]. Although it would seem likely that persistence and/or adherence to $\mathrm{OAB}$ medications among nursing home residents would be high, given the extensive nursing and supportive care, discontinuation of antimuscarinics and the factors associated with antimuscarinic discontinuation have not been examined in this setting.

Given the high frequency of UI/OAB in nursing home populations and the associated high clinical and economic burden, understanding treatment discontinuation in nursing homes may help improve adherence and quality of care for patients with OAB. Therefore, the objective of the present study was to examine treatment patterns and to characterize antimuscarinic discontinuation in long-term nursing home (LTNH) residents with OAB.

\section{METHODS}

\section{Data Source}

The Minimum Data Set (MDS) linking 2013-2015 Medicare claims data involving Part A, B and D claims $[17,18]$ was used to examine treatment patterns and predictors of antimuscarinic discontinuation among LTNH residents with OAB. The MDS is a federally mandated nursing home health assessment tool for all residents in Medicare/Medicaid-certified nursing facilities. It is administered to all nursing home residents within 14 days of admission and at quarterly intervals thereafter. It captures detailed information on physical, psychologic and psychosocial functioning and active clinical diagnosis, health conditions, treatments and services. It also provides information on patient's cognitive functioning and behavioral problems. Medicare Part A covers hospital care, initial care in skilled nursing facilities, hospice care and home health care. Part B covers services such as laboratory, ambulance, outpatient mental health and other physician services that are not included in Part A. Each Part A and Part $\mathrm{B}$ record contains up to ten diagnoses recorded according to International Classification of Diseases, Ninth Revision, Clinical Modification (ICD-9-CM) codes. Part D, launched in 2006, covers prescription benefits for Medicare beneficiaries. Each Part D Prescription Drug Event claim comprises information on prescription drug fills, including drug name, fill date, days of supply and quantity.

The study protocol was approved by the University of Houston Committee for the Protection of Human Subjects. Informed consent was not required given the deidentified nature of the data and feasibility considerations for a large retrospective database such as Medicare.

\section{Study Design and Population}

The retrospective cohort consisted of LTNH residents diagnosed with $\mathrm{OAB}$. LTNH residents were defined based on a previously validated MDS/Skilled Nursing Facility algorithm as residents with at least one nursing home episode lasting at least 101 consecutive days [19]. Patients were included in the cohort using a step-wise process if they met the following criteria: (1) $\geq 65$ years of age at index date, which was defined as the first long-stay nursing home admission date; (2) at least one claim with an $\mathrm{OAB}$ diagnosis either during the nursing home episode or prior (identified based on previously published lists of ICD-9-CM codes) [12, 20, 21] or at least one prescription claim for OABspecific medication (antimuscarinics, mirabegron or onabotulinumtoxinA specific for bladder usage) during nursing home stay (Supplementary Table 1); (3) Parts A, B and D coverage in the 3 months before the nursing home admission and until the end of the nursing home stay; (4) newly initiated antimuscarinic medication claims lasting at least 30 days continuously during nursing home stay. 


\section{Outcome Measures}

Antimuscarinics initiated during the nursing home stay were used to examine treatment patterns. Antimuscarinic medications were operationally defined using the American Hospital Formulary Service classification system and identified using National Drug Codes (NDC) [22]. Antimuscarinic medications included darifenacin, fesoterodine, oxybutynin, solifenacin, tolterodine and trospium. Treatment discontinuation was defined as the treatment gap of $\geq 30$ days between prescription refills prior to nursing home discharge date $[23,24]$. Accordingly, patients were categorized into two groups: those who discontinued the index antimuscarinic medication and those who did not discontinue the index antimuscarinic medication [25].

\section{Conceptual Framework}

A multivariable Cox proportional hazards $(\mathrm{PH})$ regression analysis was performed to assess the time to discontinuation of antimuscarinic medication among $\mathrm{OAB}$ patients. The Andersen Behavioral Model (ABM) was used to guide selection of independent variables in the analysis [26, 27]. This model postulates that an individual's use of health services is a function of predisposing, enabling and need characteristics $[26,27]$. Variables corresponding to these factors were selected based on the published literature and data available in the Medicare claims and the MDS [11, 28]. Predisposing factors are the characteristics of an individual that were in existence before the illness and included age, gender, race/ethnicity and marital status. Enabling factors are related to the ability of an individual to secure the healthcare services and included region, Medicare-Medicaid dual eligibility and urban/rural area. Urban/rural area was captured using MDS as the data source. The urban/rural distinction was based on the location of nursing home facility. The need factors describe the perceived and actual health status of an individual. The need variables were captured from both the Medicare claims and the MDS. These included index antimuscarinic agent, comorbidities measured using the Elixhauser comorbidity scores [29] and falls, prescription medication use (such as antidepressants, diuretics, beta-blockers, calcium channel blockers, anticonvulsants, angiotensin converting enzyme [ACE] inhibitors, antipsychotics, antiparkinson agents and alphablockers), anticholinergic burden (measured using the Anticholinergic Cognitive Burden [ACB] scale [30]) and body mass index (BMI). All variables were measured 3 months prior to the first long-stay nursing home admission date. Need characteristics for the ABM were also determined based on the MDS assessment recorded at nursing home admission. These included the presence of urinary or bowel incontinence (defined as always continent, occasionally incontinent, frequently incontinent, always incontinent or not rated, the former of which has been used to characterize patients with urinary incontinence in several studies [31]), bladder continence management (i.e., use of an indwelling/external catheter, ostomy and/or intermittent catheterization), urinary toileting program, response to toileting program, current toileting program or trial, depressed mood indicator and scores on the Cognitive Performance Scale (CPS) [32] and Activities of Daily Living (ADL) scale [33]. Using the CPS, a higher score is indicative of greater impairment, ranging from 0 (intact cognition) to 6 (severe cognitive impairment). Mild cognitive impairment is associated with a score of 2. A higher score on the ADL is indicative of greater impairment/dependence [33]. Using the $\mathrm{ADL}$, an individual's independence is graded as dependent [4-6], limited/extensive assistance [7-9] and independent [10-18]. Due to the higher number of missing values, UI-specific measures (i.e., use of an indwelling/external catheter, ostomy, intermittent catheterization) were not included in the multivariable Cox $\mathrm{PH}$ regression model.

\section{Statistical Analysis}

Descriptive statistics were used to characterize LTNH residents with $\mathrm{OAB}$ and antimuscarinic use. For the study cohort, the discontinuation 
rate based on the index antimuscarinic medication was reported. Categorical and continuous patient characteristics were compared among patients who discontinued the index antimuscarinic medication versus those who did not discontinue, using chi-square and student's t-tests, respectively. Time to discontinuation between different antimuscarinic users was compared using Kaplan-Meier curves and log-rank test. Proportionality hazards assumption was confirmed using the Schoenfeld test. Multivariable Cox proportional hazards regression analysis was performed to assess the risk of discontinuation among different antimuscarinic medications with time to discontinuation as the dependent variable. Independent variables were predisposing, enabling and need characteristics based on the ABM framework. Death and loss to follow-up due to lack of insurance coverage were considered as censoring criteria. All relevant variables available in the data set were included in the model.

All analyses were performed using SAS version 9.4 (SAS Institute Inc., Cary, NC) with statistical significance set at an a priori- specified level of 0.05 .

\section{RESULTS}

\section{Study Population}

Figure 1 describes the process of cohort derivation. A total of 250,016 LTNH residents $\geq 65$ years of age with evidence of $\mathrm{OAB}$ and continuous enrollment were initially identified. From this group, 11,012 (4.4\%) LTNH residents were newly initiated with antimuscarinic prescription for $\mathrm{OAB}$ and had continuous supply of medication for at least 30 days dispensed during the LTNH stay. The mean $( \pm \mathrm{SD})$ age of the cohort was $81.6( \pm 8.5)$ years. The cohort consisted of $74.6 \%$ women and $89.8 \%$ non-Hispanic whites. Most of the patients were from South (37.4\%) and Midwest $(33.5 \%)$ regions and resided in urban areas $(70.2 \%)$. Frequently reported comorbidities included hypertension (80.4\%), cardiac arrhythmias (40.7\%), diabetes (37.6\%) and fluid and electrolyte disturbances (33.4\%). The most frequently prescribed medications were antidepressants $(47.4 \%)$, beta-blockers $(41.5 \%)$ and diuretics $(36.3 \%)$. The mean $( \pm$ SD) ACB score of the cohort pre-index was $0.7( \pm 1.4)$. This was a pre-index measure of anticholinergic burden. Additional characteristics of the study cohort are summarized in Table 1 . The mean duration of nursing home stay was $530.1( \pm 268.4)$ days.

Admission MDS assessments of LTNH residents with $\mathrm{OAB}$ revealed that $23.5 \%$ had UI frequently (i.e., incontinent seven or more episodes over a 7 -day look-back period) and 37.1\% had bowel continence constantly. Data on UI were missing for $22.2 \%$. Approximately twothirds $(66.8 \%)$ of patients did not require any bladder continence management and $11.1 \%$ required some kind of continence management-including an indwelling/external catheter $(9.7 \%)$, ostomy $(1.9 \%)$ and intermittent catheterization $(0.3 \%)$. Approximately $10.1 \%$ of the patients required a urinary toileting program (i.e., scheduled training, prompted voiding and bladder training). However, only $1.1 \%$ were completely dry (i.e., continent) or showed decreased wetness (i.e., whereby urinary incontinence frequently decreased) following the toileting program. The majority of patients had intact cognition $(88.3 \%)$ but just over half $(51.1 \%)$ were dependent on their ADLs (Table 1).

\section{OAB Treatment and Antimuscarinic Discontinuation}

About $4.4 \%(n=11,012)$ of the LTNH residents were newly initiated with antimuscarinics with supply lasting at least 30 days continuously. Oxybutynin (69.8\%) was the most commonly prescribed antimuscarinic, followed by tolterodine (13.0\%) and solifenacin (12.3\%) (Table 2). About $67 \%(n=7319)$ of the study cohort was identified to have discontinued the index antimuscarinic medication during their nursing home stay, and while the median days to discontinuation was 61 (interquartile range [IQR]: 31-121) days, the mean (standard deviation) days to discontinuation was 99.9 ( \pm 105.8) days. Discontinuation rate based on the index medication in the order were darifenacin 


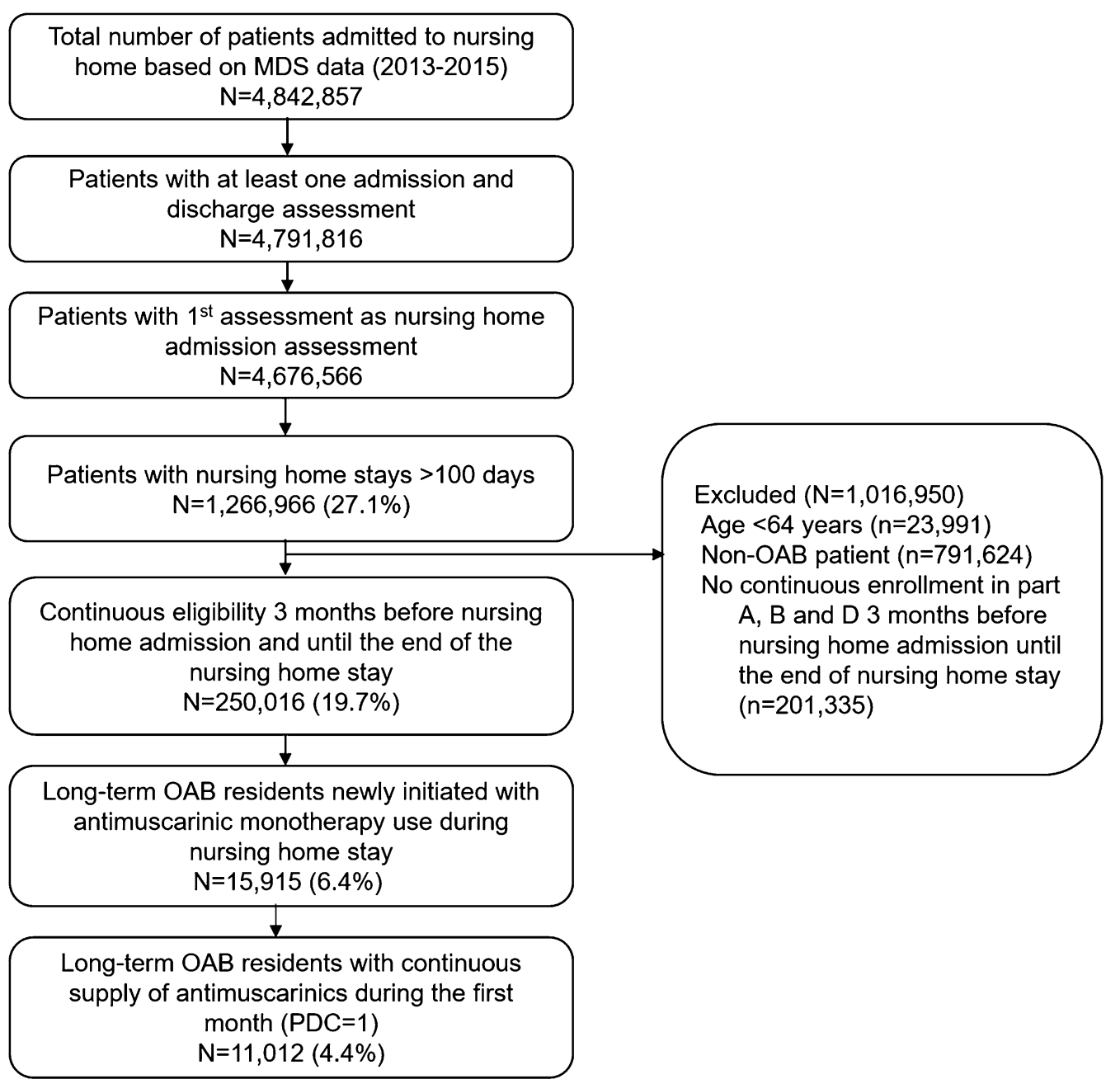

Fig. 1 Study cohort derivation. $M D S$ minimum data set, $O A B$ overactive bladder, $P D C$ Proportion Days Covered

(78.8\%), trospium $(78.2 \%)$, tolterodine $(70.8 \%)$, oxybutynin $(66.4 \%)$, fesoterodine $(61.4 \%)$ and solifenacin $(59.8 \%)$ (Table 2$)$.

The Kaplan-Meier curve with discontinuation probabilities of antimuscarinic medication over time is shown in Fig. 2. Discontinuation of antimuscarinics varied solely based on the need characteristics (Table 3). Results from the multivariable Cox $\mathrm{PH}$ regression model revealed that LTNH residents who initiated with solifenacin (Hazards Ratio (HR) 0.79, 95\% CI $0.73-0.85$ ) and darifenacin (HR 0.82, 95\% CI $0.68-0.99)$ were less likely to discontinue medication, while patients who started with tolterodine (HR 1.11, 95\% CI 1.03-1.18), darifenacin (HR 1.33, 95\% CI 1.06-1.67) and trospium (HR
1.44, 95\% CI 1.24-1.66) were more likely to discontinue medication compared to those who were started with oxybutynin. Patients who had liver disease (HR 0.82, 95\% CI 0.71-0.94), antipsychotic use (HR 0.93, 95\% CI 0.86-0.99) and participated in a urinary toileting program (HR 0.75, 95\% CI 0.58-0.97) were less likely to discontinue antimuscarinics than their counterparts, whereas LTNH residents with severe cognitive impairment (HR 1.25, 95\% CI 1.10-1.42), dependent on ADL (HR 1.13, 95\% CI 1.04-1.23) and occasionally bowel incontinent (HR 1.09, 95\% CI 1.01-1.19) were more likely to discontinue antimuscarinics compared to their counterparts. For every unit increase in the pre-index ACB burden, there was a $4 \%$ 
Table 1 Demographics of long-term nursing home residents with OAB, overall and by discontinuation status

\begin{tabular}{|c|c|c|c|c|c|c|c|}
\hline \multirow[t]{2}{*}{ Characteristic } & \multicolumn{2}{|c|}{$\begin{array}{l}\text { Total } \\
(11,012)\end{array}$} & \multicolumn{2}{|c|}{$\begin{array}{l}\text { Discontinued } \\
(7,319,66.46 \%)\end{array}$} & \multicolumn{2}{|c|}{$\begin{array}{l}\text { Not discontinued } \\
(3,693,33.54 \%)\end{array}$} & \multirow[t]{2}{*}{$P$ value } \\
\hline & $n$ & Percent (\%) & $n$ & Percent (\%) & $n$ & Percent (\%) & \\
\hline \multicolumn{8}{|l|}{ Predisposing factors } \\
\hline Age $($ mean $\pm S D)$ & 81.57 & \pm 8.52 & 81.5 & \pm 8.49 & 81.7 & \pm 8.56 & 0.313 \\
\hline $65-75$ years & 2621 & $23.80 \%$ & 1764 & $24.10 \%$ & 857 & $23.21 \%$ & 0.568 \\
\hline $75-85$ years & 3875 & $35.19 \%$ & 2570 & $35.11 \%$ & 1305 & $35.34 \%$ & \\
\hline$\geq 85$ years & 4516 & $41.01 \%$ & 2985 & $40.78 \%$ & 1531 & $41.46 \%$ & \\
\hline \multicolumn{8}{|l|}{ Gender } \\
\hline Male & 2799 & $25.42 \%$ & 1877 & $25.65 \%$ & 922 & $24.97 \%$ & 0.44 \\
\hline Female & 8213 & $74.58 \%$ & 5442 & $74.35 \%$ & 2771 & $75.03 \%$ & \\
\hline \multicolumn{8}{|l|}{ Race/ethnicity } \\
\hline Non-Hispanic White & 9891 & $89.82 \%$ & 6540 & $89.36 \%$ & 3351 & $90.74 \%$ & 0.139 \\
\hline Non-Hispanic Black & 734 & $6.67 \%$ & 514 & $7.02 \%$ & 220 & $5.96 \%$ & \\
\hline Hispanics & 123 & $1.12 \%$ & 83 & $1.13 \%$ & 40 & $1.08 \%$ & \\
\hline Others & 264 & $2.40 \%$ & 182 & $2.49 \%$ & 82 & $2.22 \%$ & \\
\hline Marital status & & & & & & & 0.82 \\
\hline Unmarried (single/widowed) & 8415 & $76.42 \%$ & 5580 & $76.24 \%$ & 2,835 & $76.77 \%$ & \\
\hline Married & 2529 & $22.97 \%$ & 1694 & $23.15 \%$ & 835 & $22.61 \%$ & \\
\hline Others & 68 & $0.62 \%$ & 45 & $0.61 \%$ & 23 & $0.62 \%$ & \\
\hline \multicolumn{8}{|l|}{ Enabling factors } \\
\hline Medicare/Medicaid dual eligible & 5077 & $46.10 \%$ & 3462 & $47.30 \%$ & 1615 & $43.73 \%$ & 0.004 \\
\hline Region & & & & & & & 0.39 \\
\hline South & 4123 & $37.44 \%$ & 2778 & $37.96 \%$ & 1345 & $36.42 \%$ & \\
\hline Northeast & 2057 & $18.68 \%$ & 1369 & $18.70 \%$ & 688 & $18.63 \%$ & \\
\hline Midwest & 3688 & $33.49 \%$ & 2420 & $33.06 \%$ & 1268 & $34.34 \%$ & \\
\hline West & 1144 & $10.39 \%$ & 752 & $10.27 \%$ & 392 & $10.61 \%$ & \\
\hline Urban-rural & & & & & & & 0.377 \\
\hline Rural & 3283 & $29.81 \%$ & 2,162 & $29.54 \%$ & 1121 & $30.35 \%$ & \\
\hline Urban & 7729 & $70.19 \%$ & 5157 & $70.46 \%$ & 2572 & $69.65 \%$ & \\
\hline
\end{tabular}


Table 1 continued

\begin{tabular}{|c|c|c|c|c|c|c|c|}
\hline \multirow[t]{2}{*}{ Characteristic } & \multicolumn{2}{|c|}{$\begin{array}{l}\text { Total } \\
(11,012)\end{array}$} & \multicolumn{2}{|c|}{$\begin{array}{l}\text { Discontinued } \\
(7,319,66.46 \%)\end{array}$} & \multicolumn{2}{|c|}{$\begin{array}{l}\text { Not discontinued } \\
(3,693,33.54 \%)\end{array}$} & \multirow[t]{2}{*}{$P$ value } \\
\hline & $\bar{n}$ & Percent (\%) & $n$ & Percent (\%) & $\bar{n}$ & Percent (\%) & \\
\hline \multicolumn{8}{|l|}{ Need factors } \\
\hline Index antimuscarinic prescription $¥$ & & & & & & & $<0.0001$ \\
\hline Solifenacin & 1353 & $12.29 \%$ & 809 & $11.05 \%$ & 544 & $14.73 \%$ & \\
\hline Fesoterodin & 189 & $1.72 \%$ & 116 & $1.58 \%$ & 73 & $1.98 \%$ & \\
\hline Tolterodine & 1437 & $13.05 \%$ & 1017 & $13.90 \%$ & 420 & $11.37 \%$ & \\
\hline Trospium & 243 & $2.21 \%$ & 190 & $2.60 \%$ & 53 & $1.44 \%$ & \\
\hline Darifenacin & 99 & $0.90 \%$ & 78 & $1.07 \%$ & 21 & $0.57 \%$ & \\
\hline Oxybutinine & 7691 & $69.84 \%$ & 5109 & $69.80 \%$ & 2582 & $69.92 \%$ & \\
\hline \multicolumn{8}{|l|}{ Elixhauser comorbidities } \\
\hline Elixhauser Index Score (mean \pm SD) & 10.12 & \pm 8.77 & 9.8 & \pm 8.67 & 10.7 & \pm 8.95 & $<0.0001$ \\
\hline Prior history of falls & 2858 & $25.95 \%$ & 1833 & $25.04 \%$ & 1025 & $27.76 \%$ & 0.003 \\
\hline Congestive heart failure & 3171 & $28.80 \%$ & 2040 & $27.87 \%$ & 1131 & $30.63 \%$ & 0.0001 \\
\hline Cardiac arrhythmias & 4477 & $40.66 \%$ & 2,882 & $39.38 \%$ & 1595 & $43.19 \%$ & $<0.0001$ \\
\hline Valvular disease & 1715 & $15.57 \%$ & 1068 & $14.59 \%$ & 647 & $17.52 \%$ & 0.001 \\
\hline Pulmonary circulation disorders & 683 & $6.20 \%$ & 414 & $5.66 \%$ & 269 & $7.28 \%$ & 0.407 \\
\hline Peripheral vascular disorders & 2747 & $24.95 \%$ & 1808 & $24.70 \%$ & 939 & $25.43 \%$ & 0.788 \\
\hline Hypertension & 8851 & $80.38 \%$ & 5888 & $80.45 \%$ & 2963 & $80.23 \%$ & 0.732 \\
\hline Paralysis & 635 & $5.77 \%$ & 426 & $5.82 \%$ & 209 & $5.66 \%$ & 0.568 \\
\hline Other neurologic disorders & 2780 & $25.25 \%$ & 1860 & $25.41 \%$ & 920 & $24.91 \%$ & 0.248 \\
\hline Chronic pulmonary disease & 3404 & $30.91 \%$ & 2236 & $30.55 \%$ & 1168 & $31.63 \%$ & 0.469 \\
\hline Diabetes & 4137 & $37.57 \%$ & 2767 & $37.81 \%$ & 1370 & $37.10 \%$ & 0.615 \\
\hline Hypothyroidism & 2643 & $24.00 \%$ & 1746 & $23.86 \%$ & 897 & $24.29 \%$ & 0.195 \\
\hline Renal failure & 2031 & $18.44 \%$ & 1325 & $18.10 \%$ & 706 & $19.12 \%$ & 0.003 \\
\hline Liver disease & 357 & $3.24 \%$ & 211 & $2.88 \%$ & 146 & $3.95 \%$ & 0.505 \\
\hline Peptic ulcer & 164 & $1.49 \%$ & 105 & $1.43 \%$ & 59 & $1.60 \%$ & 0.127 \\
\hline AIDS/HIV & 14 & $0.13 \%$ & $* * *$ & $* * *$ & $* * *$ & $* * *$ & 0.58 \\
\hline Lymphoma & 91 & $0.83 \%$ & 58 & $0.79 \%$ & 33 & $0.89 \%$ & 0.0002 \\
\hline Metastatic cancer & 163 & $1.48 \%$ & 86 & $1.18 \%$ & 77 & $2.09 \%$ & 0.141 \\
\hline Solid tumor without metastasis & 782 & $7.10 \%$ & 501 & $6.85 \%$ & 281 & $7.61 \%$ & 0.576 \\
\hline Rheumatoid arthritis & 622 & $5.65 \%$ & 407 & $5.56 \%$ & 215 & $5.82 \%$ & 0.339 \\
\hline Coagulopathy & 510 & $4.63 \%$ & 329 & $4.50 \%$ & 181 & $4.90 \%$ & 0.943 \\
\hline
\end{tabular}


Table 1 continued

\begin{tabular}{|c|c|c|c|c|c|c|c|}
\hline \multirow[t]{2}{*}{ Characteristic } & \multicolumn{2}{|c|}{$\begin{array}{l}\text { Total } \\
(11,012)\end{array}$} & \multicolumn{2}{|c|}{$\begin{array}{l}\text { Discontinued } \\
(7,319,66.46 \%)\end{array}$} & \multicolumn{2}{|c|}{$\begin{array}{l}\text { Not discontinued } \\
(3,693,33.54 \%)\end{array}$} & \multirow[t]{2}{*}{$P$ value } \\
\hline & $n$ & Percent (\%) & $n$ & Percent (\%) & $n$ & Percent (\%) & \\
\hline Obesity & 847 & $7.69 \%$ & 562 & $7.68 \%$ & 285 & $7.72 \%$ & 0.214 \\
\hline Weight loss & 962 & $8.74 \%$ & 622 & $8.50 \%$ & 340 & $9.21 \%$ & 0.102 \\
\hline Fluid and electrolyte disorders & 3682 & $33.44 \%$ & 2,409 & $32.91 \%$ & 1273 & $34.47 \%$ & 0.937 \\
\hline Blood loss anemias & 222 & $2.02 \%$ & 147 & $2.01 \%$ & 75 & $2.03 \%$ & 0.299 \\
\hline Deficiency anemias & 1020 & $9.26 \%$ & 663 & $9.06 \%$ & 357 & $9.67 \%$ & 0.349 \\
\hline Alcohol abuse & 185 & $1.68 \%$ & 117 & $1.60 \%$ & 68 & $1.84 \%$ & 0.953 \\
\hline Drug abuse & 189 & $1.72 \%$ & 126 & $1.72 \%$ & 63 & $1.71 \%$ & 0.266 \\
\hline Psychoses & 1905 & $17.30 \%$ & 1287 & $17.58 \%$ & 618 & $16.73 \%$ & 0.005 \\
\hline Depression & 3171 & $28.80 \%$ & 2171 & $29.66 \%$ & 1000 & $27.08 \%$ & 0.018 \\
\hline \multicolumn{8}{|l|}{ Medication use } \\
\hline Alpha-blockers & 228 & $2.07 \%$ & 155 & $2.12 \%$ & 73 & $1.98 \%$ & 0.624 \\
\hline Beta-blockers & 4564 & $41.45 \%$ & 3030 & $41.40 \%$ & 1534 & $41.54 \%$ & 0.889 \\
\hline Calcium channel blockers & 2984 & $27.10 \%$ & 2034 & $27.79 \%$ & 950 & $25.72 \%$ & 0.021 \\
\hline ACE inhibitors & 2985 & $27.11 \%$ & 1991 & $27.20 \%$ & 994 & $26.92 \%$ & 0.749 \\
\hline Diuretics & 3996 & $36.29 \%$ & 2632 & $35.96 \%$ & 1364 & $36.93 \%$ & 0.316 \\
\hline Antidepressants & 5219 & $47.39 \%$ & 3543 & $48.41 \%$ & 1676 & $45.38 \%$ & 0.003 \\
\hline Antipsychotics & 1716 & $15.58 \%$ & 1,170 & $15.99 \%$ & 546 & $14.78 \%$ & 0.101 \\
\hline Anticonvulsants & 2742 & $24.90 \%$ & 1,884 & $25.74 \%$ & 858 & $23.23 \%$ & 0.004 \\
\hline Antiparkinson agents & 1130 & $10.26 \%$ & 759 & $10.37 \%$ & 371 & $10.05 \%$ & 0.597 \\
\hline $\mathrm{ACB}$ scale $($ mean $\pm \mathrm{SD})$ & 0.65 & \pm 1.35 & 0.72 & \pm 1.42 & 0.53 & \pm 1.18 & $<0.0001$ \\
\hline Body mass index* & & & & & & & 0.426 \\
\hline Underweight & 2878 & $26.14 \%$ & 1940 & $26.51 \%$ & 938 & $25.40 \%$ & \\
\hline Normal weight & 482 & $4.38 \%$ & 323 & $4.41 \%$ & 159 & $4.31 \%$ & \\
\hline Overweight & 2385 & $21.66 \%$ & 1600 & $21.86 \%$ & 785 & $21.26 \%$ & \\
\hline Obese & 2686 & $24.39 \%$ & 1750 & $23.91 \%$ & 936 & $25.35 \%$ & \\
\hline Missing/unknown & 2581 & $23.44 \%$ & 1706 & $23.31 \%$ & 875 & $23.69 \%$ & \\
\hline Urinary continence ${ }^{*} \dagger$ & & & & & & & $<0.0001$ \\
\hline Always continent & 1835 & $16.66 \%$ & 1153 & $15.75 \%$ & 682 & $18.47 \%$ & \\
\hline Occasionally incontinent & 1932 & $17.54 \%$ & 1216 & $16.61 \%$ & 716 & $19.39 \%$ & \\
\hline Frequently incontinent & 2583 & $23.46 \%$ & 1755 & $23.98 \%$ & 828 & $22.42 \%$ & \\
\hline Always incontinent & 1,241 & $11.27 \%$ & 941 & $12.86 \%$ & 300 & $8.12 \%$ & \\
\hline
\end{tabular}


Table 1 continued

\begin{tabular}{|c|c|c|c|c|c|c|c|}
\hline \multirow[t]{2}{*}{ Characteristic } & \multicolumn{2}{|c|}{$\begin{array}{l}\text { Total } \\
(11,012)\end{array}$} & \multicolumn{2}{|c|}{$\begin{array}{l}\text { Discontinued } \\
(7,319,66.46 \%)\end{array}$} & \multicolumn{2}{|c|}{$\begin{array}{l}\text { Not discontinued } \\
(3,693,33.54 \%)\end{array}$} & \multirow[t]{2}{*}{$P$ value } \\
\hline & $n$ & Percent (\%) & $n$ & Percent (\%) & $n$ & Percent (\%) & \\
\hline Not rated & 975 & $8.85 \%$ & 644 & $8.80 \%$ & 331 & $8.96 \%$ & \\
\hline Missing/unknown & 2446 & $22.21 \%$ & 1610 & $22.00 \%$ & 836 & $22.64 \%$ & \\
\hline \multicolumn{8}{|l|}{ Bladder continence management* } \\
\hline Indwelling/external catheter & & & & & & & 0.663 \\
\hline Yes & 1068 & $9.70 \%$ & 703 & $9.61 \%$ & 365 & $9.88 \%$ & \\
\hline No & 7514 & $68.23 \%$ & 5015 & $68.52 \%$ & 2499 & $67.67 \%$ & \\
\hline Missing/unknown & 2430 & $22.07 \%$ & 1601 & $21.87 \%$ & 829 & $22.45 \%$ & \\
\hline Ostomy & & & & & & & 0.321 \\
\hline Yes & 210 & $1.91 \%$ & 149 & $2.04 \%$ & 61 & $1.65 \%$ & \\
\hline No & 8372 & $76.03 \%$ & 5569 & $76.09 \%$ & 2803 & $75.90 \%$ & \\
\hline Missing/unknown & 2,430 & $22.07 \%$ & 1601 & $21.87 \%$ & 829 & $22.45 \%$ & \\
\hline Intermittent catheterization & & & & & & & 0.327 \\
\hline Yes & 34 & $0.31 \%$ & 19 & $0.26 \%$ & 15 & $0.41 \%$ & \\
\hline No & 8548 & $77.62 \%$ & 5699 & $77.87 \%$ & 2,849 & $77.15 \%$ & \\
\hline Missing/unknown & 2430 & $22.07 \%$ & 1601 & $21.87 \%$ & 829 & $22.45 \%$ & \\
\hline None of the above bladder appliance & & & & & & & 0.766 \\
\hline Yes & 7359 & $66.83 \%$ & 4907 & $67.04 \%$ & 2452 & $66.40 \%$ & \\
\hline No & 1223 & $11.11 \%$ & 811 & $11.08 \%$ & 412 & $11.16 \%$ & \\
\hline Missing/unknown & 2430 & $22.07 \%$ & 1601 & $21.87 \%$ & 829 & $22.45 \%$ & \\
\hline Urinary toileting program* & & & & & & & $<0.0001$ \\
\hline Yes & 1115 & $10.13 \%$ & 795 & $10.86 \%$ & 320 & $8.67 \%$ & \\
\hline No & 6020 & $54.67 \%$ & 4170 & $56.97 \%$ & 1850 & $50.09 \%$ & \\
\hline Missing/unknown & 3877 & $35.21 \%$ & 2354 & $32.16 \%$ & 1,523 & $41.24 \%$ & \\
\hline Response to toileting program* & & & & & & & 0.096 \\
\hline No improvement & 151 & $1.37 \%$ & 111 & $1.52 \%$ & 40 & $1.08 \%$ & \\
\hline Decreased wetness & 81 & $0.74 \%$ & 61 & $0.83 \%$ & 20 & $0.54 \%$ & \\
\hline Completely dry (continent) & 39 & $0.35 \%$ & 26 & $0.36 \%$ & 13 & $0.35 \%$ & \\
\hline Missing/unknown & 10,741 & $97.54 \%$ & 7121 & $97.29 \%$ & 3620 & $98.02 \%$ & \\
\hline Current toileting program or trial* & & & & & & & 0.002 \\
\hline Yes & 751 & $6.82 \%$ & 528 & $7.21 \%$ & 223 & $6.04 \%$ & \\
\hline No & 515 & $4.68 \%$ & 368 & $5.03 \%$ & 147 & $3.98 \%$ & \\
\hline Missing/unknown & 9746 & $88.50 \%$ & 6423 & $87.76 \%$ & 3323 & $89.98 \%$ & \\
\hline
\end{tabular}


Table 1 continued

\begin{tabular}{|c|c|c|c|c|c|c|c|}
\hline \multirow[t]{2}{*}{ Characteristic } & \multicolumn{2}{|c|}{$\begin{array}{l}\text { Total } \\
(11,012)\end{array}$} & \multicolumn{2}{|c|}{$\begin{array}{l}\text { Discontinued } \\
(7,319,66.46 \%)\end{array}$} & \multicolumn{2}{|c|}{$\begin{array}{l}\text { Not discontinued } \\
(3,693,33.54 \%)\end{array}$} & \multirow[t]{2}{*}{$P$ value } \\
\hline & $n$ & Percent (\%) & $n$ & Percent (\%) & $n$ & Percent (\%) & \\
\hline Bowel continence* & & & & & & & $<0.0001$ \\
\hline Always continent & 4090 & $37.14 \%$ & 2574 & $35.17 \%$ & 1516 & $41.05 \%$ & \\
\hline Occasionally incontinent & 1216 & $11.04 \%$ & 792 & $10.82 \%$ & 424 & $11.48 \%$ & \\
\hline Frequently incontinent & 1638 & $14.87 \%$ & 1158 & $15.82 \%$ & 480 & $13.00 \%$ & \\
\hline Always incontinent & 1416 & $12.86 \%$ & 1046 & $14.29 \%$ & 370 & $10.02 \%$ & \\
\hline Not rated & 206 & $1.87 \%$ & 138 & $1.89 \%$ & 68 & $1.84 \%$ & \\
\hline Missing/unknown & 2446 & $22.21 \%$ & 1611 & $22.01 \%$ & 835 & $22.61 \%$ & \\
\hline MDS Cognition Scale* & & & & & & & $<0.0001$ \\
\hline Intact & 9724 & $88.30 \%$ & 6391 & $87.32 \%$ & 3333 & $90.25 \%$ & \\
\hline Mild & 146 & $1.33 \%$ & 93 & $1.27 \%$ & 53 & $1.44 \%$ & \\
\hline Moderate & 370 & $3.36 \%$ & 241 & $3.29 \%$ & 129 & $3.49 \%$ & \\
\hline Moderate/severe & 93 & $0.84 \%$ & 69 & $0.94 \%$ & 24 & $0.65 \%$ & \\
\hline Severe & 395 & $3.59 \%$ & 311 & $4.25 \%$ & 84 & $2.27 \%$ & \\
\hline Missing/unknown & 284 & $2.58 \%$ & 214 & $2.92 \%$ & 70 & $1.90 \%$ & \\
\hline Activities of daily living* & & & & & & & $<0.0001$ \\
\hline Independent & 2708 & $24.59 \%$ & 1578 & $21.56 \%$ & 1130 & $30.60 \%$ & \\
\hline Limited/extensive assistance & 379 & $3.44 \%$ & 258 & $3.53 \%$ & 121 & $3.28 \%$ & \\
\hline Dependent & 5624 & $51.07 \%$ & 3981 & $54.39 \%$ & 1643 & $44.49 \%$ & \\
\hline Missing/unknown & 2301 & $20.90 \%$ & 1502 & $20.52 \%$ & 799 & $21.64 \%$ & \\
\hline Depressed mood indicator* & & & & & & & $<0.0001$ \\
\hline Yes & 245 & $2.22 \%$ & 182 & $2.49 \%$ & 63 & $1.71 \%$ & \\
\hline No & 7637 & $69.35 \%$ & 5,135 & $70.16 \%$ & 2,502 & $67.75 \%$ & \\
\hline Missing/unknown & 3130 & $28.42 \%$ & 2002 & $27.35 \%$ & 1128 & $30.54 \%$ & \\
\hline
\end{tabular}

$A C B$ anticholinergic burden, $A I D S / H I V$ acquired immune deficiency syndrome/human immune deficiency virus, $L T N H$ long-term nursing home, $M D S$ minimum data set, $O A B$ overactive bladder, $S D$ standard deviation

Significant values are bolded

*Based on MDS Admission Assessment and includes missing data

**Based on enrollment data and includes missing data

${ }^{* * *}$ Cannot report due to small cell size

$\dagger$ Categories of urinary incontinence were defined as: always continent (over a 7-day look-back period, resident was continent with urine, with no episodes of incontinence); occasionally incontinent (over a 7-day look-back period, resident was incontinent less than 7 episodes); frequently incontinent (over a 7-day look-back period, resident was incontinent 7 or more episodes); always incontinent (over a 7-day look-back period, resident had no continent episodes); not rated (over a 7-day look-back period the resident had an indwelling catheter, condom catheter, ostomy or no urine output for the entire 7 days)

$¥$ Index antimuscarinic prescription refers to antimuscarinic medication that patients were newly started with upon nursing home admission 
increased likelihood of discontinuing the antimuscarinic medication.

\section{DISCUSSION}

To our knowledge, this is the first study to characterize discontinuation among different antimuscarinics in LTNH residents with OAB. A total of $11,012(4.4 \%)$ residents were newly started with an $\mathrm{OAB}$ antimuscarinic prescription during the LTNH stay. Despite the significant impact of $\mathrm{OAB}$ on patients' quality of life and the availability of effective pharmacotherapies that can mitigate those symptoms, a small portion elderly LTNH residents in the current study initiated antimuscarinic medications. The low proportion of patients initiating pharmacotherapies for their $\mathrm{OAB}$ identified in the present study may be due to several factors including prescription costs and coverage, perceptions around antimuscarinic safety and effectiveness, inclusion of high-level anticholinergics as part of Beers criteria, patient and provider preferences as well as low rates of persistence with $\mathrm{OAB}$ medications [24, 34].

Consistent with the previous US-based literature [35-37], the most commonly prescribed antimuscarinics observed in the present study were oxybutynin $(69.8 \%)$ followed by solifenacin $(12.3 \%)$ and tolterodine $(13.0 \%)$. The high percentage of oxybutynin use is consistent with Zarowitz et al. who reported that among US nursing facilities, $61.1 \%$ were treated with oxybutynin extended release [7]. The high frequency of oxybutynin prescriptions may likely be because of generic status, formulary coverage and capitated payment for initial nursing home stays. High rates of use of oxybutynin may be another example of potentially inappropriate medication use within nursing homes, especially in older populations with cognitive issues $[38$, 39]. In fact, according to the FORTA list, oxybutynin is rated as $\mathrm{C} / \mathrm{D}$ and should be used with caution or even avoided in older populations [40]. Therefore, if behavioral options are not effective and pharmacotherapies are required given the risk of cognitive effects associated with oxybutynin, caution must be exercised prior to prescribing oxybutynin in older and/or frail adults presenting with $\mathrm{OAB}$ [41-44]. The high rates of oxybutynin use combined with relatively longer times to discontinuation identified in this study give reasons for concern. Although physicians were not queried in this study, it may indicate that clinicians are possibly complacent regarding these risks, especially for the older populations with cognitive issues.

As $\mathrm{OAB}$ usually requires long-term treatment to achieve symptom control, persistence to prescribed pharmacotherapy is important in achieving the best treatment outcomes. This study found that almost $67 \%$ of the study cohort discontinued antimuscarinic medication prior to their nursing home discharge and several need factors were associated with treatment discontinuation. Across other studies, the discontinuation rate of antimuscarinics among individuals with $\mathrm{OAB}$ has been estimated to be about $43-83 \%$ based on a systematic review $[14,24]$ and $52 \%$ to $89 \%$ from a more recent study [16]. Although persistence in nursing homes may naturally be higher compared to community-dwelling individuals because of the active involvement of care providers in medication management, it is still sub-optimal given that adherence to antimuscarinics improves quality of life in patients with OAB [45]. Although patient-reported reasons for antimuscarinic discontinuation and non-adherence could not be evaluated in the present study because of the data limitations, future research on this is needed to explore the common reasons for discontinuation and develop personalized treatment plans to optimize $\mathrm{OAB}$ management.

In the current study, the specific type of index antimuscarinic medication was significantly associated with antimuscarinic discontinuation. Compared to oxybutinin users, solifenacin and fesoterodine users had less risk of discontinuation, whereas tolterodine, darifenacin and trospium carried higher risk of discontinuation. However, in a study that assessed antimuscarinic discontinuation among a Medicare elderly population in a community setting, all five agents (solifenacin, fesoterodine, darifenacin, tolterodine and trospium) showed less 
Table 2 Overall treatment pattern, discontinuation rate and time to discontinuation based on index antimuscarinic medication during long-term nursing home stays

\begin{tabular}{|c|c|c|c|c|c|c|}
\hline \multirow[b]{2}{*}{ Total } & \multicolumn{2}{|c|}{$\begin{array}{l}\text { Antimuscarinic } \\
\text { utilization rate }\end{array}$} & \multicolumn{2}{|c|}{$\begin{array}{l}\text { Antimuscarinic } \\
\text { discontinuation rate }\end{array}$} & \multicolumn{2}{|c|}{$\begin{array}{l}\text { Time to discontinuation of index } \\
\text { antimuscarinic medication }\end{array}$} \\
\hline & $\begin{array}{l}n \\
11,012\end{array}$ & $\begin{array}{l}\text { Percent (\%) } \\
100.00 \%\end{array}$ & $\begin{array}{l}n \\
7319\end{array}$ & $\begin{array}{l}\text { Percent }(\%) \\
66.46 \%\end{array}$ & Mean (SD) & Median (IQR) \\
\hline \multicolumn{7}{|c|}{ Index antimuscarinic medication } \\
\hline Oxybutinine & 7691 & 69.8 & 5109 & 66.4 & $96.98(102.19)$ & $61(31-121)$ \\
\hline Tolterodine & 1437 & 13.0 & 1017 & 70.8 & $97.85(104.01)$ & $61(31-113)$ \\
\hline Solifenacin & 1353 & 12.3 & 809 & 59.8 & $121.82(124.42)$ & $71(43-147)$ \\
\hline Trospium & 243 & 2.2 & 190 & 78.2 & $80.64(97.08)$ & $46(31-91)$ \\
\hline Fesoterodin & 189 & 1.7 & 116 & 61.4 & $122.39(121.58)$ & $75(43-151)$ \\
\hline Darifenacin & 99 & 0.9 & 78 & 78.8 & $100.59(111.14)$ & $59(32-114)$ \\
\hline
\end{tabular}

$I Q R$ interquartile range, $S D$ standard deviation

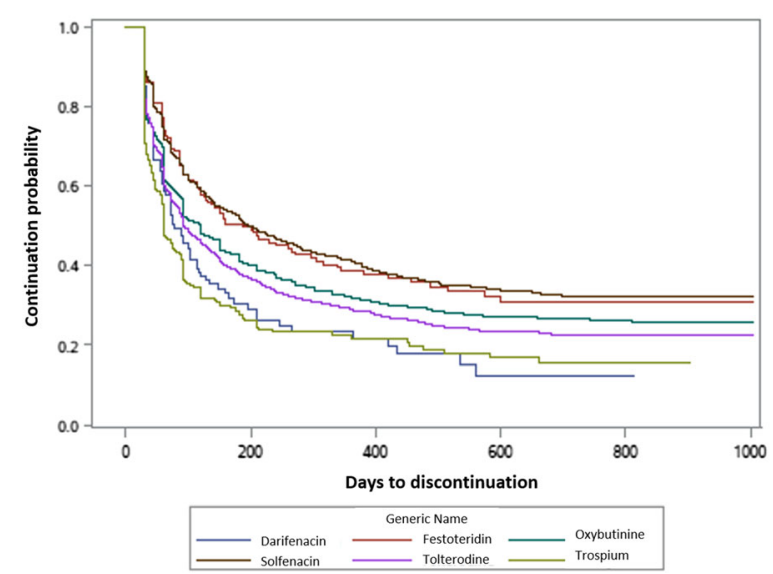

Fig. 2 Kaplan-Meier curve: discontinuation probability by type of antimuscarinic medication

risk of discontinuation compared to oxybutynin [16].

In this study, $67 \%$ of LTNH residents discontinued their index antimuscarinic, which is similar to the proportion reported by Vouri et al., among community-based Medicare older adults [16]. An unexpected finding was that none of the predisposing and enabling factors contributed to antimuscarinic discontinuation in LTNH residents, which is in contrast to the aforementioned community-based study.
Furthermore, in the Vouri et al. study patients with congestive heart failure, mild cognitive impairment, depression, diabetes and hypertension were all less likely to discontinue their antimuscarinics [16]. However, in the current study, none of these factors were associated with discontinuation. It is possible that differences between the two studies are due to underlying characteristics of the two populations. The presence of liver failure, obesity and utilization history of antipsychotics were all negatively associated with discontinuation, whereas use of calcium channel blockers/anticonvulsants and severe cognitive decline were positively associated with discontinuation. The current study indicated that a high anticholinergic burden was associated with higher likelihood of discontinuation. Although the overall anticholinergic burden was assessed pre-index in the present study, and therefore relatively low (0.53), it was nevertheless associated with increased discontinuation. As all of the antimuscarinics assessed in the present study would have had an ACB score of 3 had the anticholinergic burden been assessed posttreatment initiation, the results from the present study indicate that even low levels of anticholinergic burden impact persistence among OAB LTNH residents. It is, however, very 
Table 3 Factors associated with discontinuation of antimuscarinic drugs in long-term nursing home residents with OAB. Results from multivariable Cox proportional hazards regression model

\begin{tabular}{lll}
\hline Characteristic & Adjusted hazards ratio $(95 \% \mathrm{CI})$ & $P$ value \\
\hline
\end{tabular}

Predisposing factors

Age $($ mean $\pm S D)$

$$
\begin{aligned}
& 65-75 \text { years } \\
& 75-85 \text { years } \\
& \geq 85 \text { years }
\end{aligned}
$$

Reference

$0.96(0.90-1.02)$

0.176

$0.97(0.90-1.04)$

Gender

Male

Reference

Female

$0.95(0.89-1.00)$

Race/ethnicity

Non-Hispanic White

Reference

Non-Hispanic Black

$1.05(0.95-1.15)$

0.346

Hispanics

$0.99(0.79-1.23)$

0.906

Others

$1.04(0.89-1.21)$

0.632

Marital status

Unmarried (single/widowed)

Married

$1.04(0.98-1.10)$

0.214

Others

$1.03(0.76-1.38)$

Enabling factors

Medicare/Medicaid dual eligible

$0.98(0.93-1.03)$

Region

South

Reference

Northeast

$0.98(0.91-1.05)$

0.509

Midwest

$1.02(0.96-1.08)$

0.558

West

$1.05(0.96-1.14)$

0.271

Urban-rural

Rural

Reference

Urban

$1.03(0.98-1.09)$

0.258

\section{Need factors}

Index antimuscarinic prescription¥

Solifenacin

$0.79(0.73-0.85)$

$<0.0001$

Fesoterodine

$0.82(0.68-0.99)$

0.039

Tolterodine

$1.11(1.03-1.18)$

0.004 
Table 3 continued

\begin{tabular}{|c|c|c|}
\hline Characteristic & Adjusted hazards ratio $(95 \% \mathrm{CI})$ & $P$ value \\
\hline Trospium & $1.44(1.24-1.66)$ & $<0.0001$ \\
\hline Darifenacin & $1.33(1.06-1.67)$ & 0.012 \\
\hline Oxybutinin & Reference & \\
\hline \multicolumn{3}{|l|}{ Elixhauser comorbidities } \\
\hline \multicolumn{3}{|c|}{ Elixhauser Index score (mean $\pm S D)$} \\
\hline Prior history of falls & $0.95(0.90-1.00)$ & 0.050 \\
\hline Congestive heart failure & $0.98(0.93-1.04)$ & 0.518 \\
\hline Cardiac arrhythmias & $0.96(0.92-1.01)$ & 0.141 \\
\hline Valvular disease & $0.95(0.89-1.02)$ & 0.135 \\
\hline Pulmonary circulation disorders & $0.92(0.83-1.02)$ & 0.124 \\
\hline Peripheral vascular disorders & $0.97(0.92-1.02)$ & 0.248 \\
\hline Hypertension & $1.00(0.94-1.06)$ & 0.888 \\
\hline Paralysis & $1.01(0.91-1.11)$ & 0.908 \\
\hline Other neurologic disorders & $1.00(0.94-1.06)$ & 0.979 \\
\hline Chronic pulmonary disease & $1.01(0.95-1.06)$ & 0.829 \\
\hline Diabetes & $1.01(0.96-1.06)$ & 0.833 \\
\hline Hypothyroidism & $0.99(0.93-1.04)$ & 0.650 \\
\hline Renal failure & $1.01(0.95-1.08)$ & 0.653 \\
\hline Liver disease & $0.82(0.71-0.94)$ & 0.005 \\
\hline Peptic ulcer & $0.98(0.81-1.20)$ & 0.861 \\
\hline AIDS/HIV & $1.71(0.96-3.03)$ & 0.067 \\
\hline Lymphoma & $0.85(0.66-1.11)$ & 0.238 \\
\hline Metastatic cancer & $0.80(0.64-1.01)$ & 0.056 \\
\hline Solid tumor without metastasis & $1.03(0.94-1.14)$ & 0.492 \\
\hline Rheumatoid arthritis & $1.02(0.92-1.13)$ & 0.679 \\
\hline Coagulopathy & $1.03(0.92-1.15)$ & 0.633 \\
\hline Obesity & $1.07(0.98-1.18)$ & 0.134 \\
\hline Weight loss & $0.97(0.89-1.06)$ & 0.494 \\
\hline Fluid and electrolyte disorders & $1.00(0.95-1.05)$ & 0.852 \\
\hline Blood loss anemias & $1.06(0.90-1.25)$ & 0.495 \\
\hline Deficiency anemias & $1.00(0.92-1.08)$ & 0.924 \\
\hline Alcohol abuse & $0.97(0.80-1.17)$ & 0.757 \\
\hline
\end{tabular}


Table 3 continued

\begin{tabular}{|c|c|c|}
\hline Characteristic & Adjusted hazards ratio $(95 \% \mathrm{CI})$ & $P$ value \\
\hline Drug abuse & $0.95(0.79-1.14)$ & 0.567 \\
\hline Psychoses & $0.98(0.92-1.05)$ & 0.606 \\
\hline Depression & $1.06(1.00-1.12)$ & 0.060 \\
\hline \multicolumn{3}{|l|}{ Medication use } \\
\hline Alpha-blockers & $1.01(0.86-1.18)$ & 0.935 \\
\hline Beta-blockers & $1.00(0.96-1.06)$ & 0.872 \\
\hline Calcium channel blockers & $1.06(1.01-1.12)$ & 0.022 \\
\hline ACE inhibitors & $1.00(0.95-1.05)$ & 0.931 \\
\hline Diuretics & $0.98(0.93-1.03)$ & 0.379 \\
\hline Antidepressants & $1.00(0.95-1.05)$ & 0.896 \\
\hline Antipsychotics & $0.93(0.86-0.99)$ & 0.032 \\
\hline Anticonvulsants & $1.07(1.01-1.13)$ & 0.016 \\
\hline Antiparkinson agents & $1.00(0.92-1.08)$ & 0.945 \\
\hline $\mathrm{ACB}$ scale $($ mean $\pm \mathrm{SD})$ & $1.04(1.03-1.06)$ & $<0.0001$ \\
\hline \multicolumn{3}{|l|}{ Body mass index* } \\
\hline Underweight & Reference & \\
\hline Normal weight & $1.04(0.92-1.17)$ & 0.554 \\
\hline Overweight & $0.97(0.90-1.03)$ & 0.314 \\
\hline Obese & $0.90(0.84-0.97)$ & 0.006 \\
\hline Missing/unknown & $0.99(0.82-1.21)$ & 0.960 \\
\hline \multicolumn{3}{|l|}{ Urinary continence ${ }^{*} \dagger$} \\
\hline Always continent & Reference & \\
\hline Occasionally incontinent & $0.95(0.87-1.04)$ & 0.258 \\
\hline Frequently incontinent & $0.99(0.90-1.08)$ & 0.800 \\
\hline Always incontinent & $1.09(0.96-1.22)$ & 0.176 \\
\hline Not rated & $1.03(0.83-1.28)$ & 0.809 \\
\hline Missing/unknown & $0.65(0.28-1.51)$ & 0.315 \\
\hline \multicolumn{3}{|c|}{ Bladder continence management* } \\
\hline \multicolumn{3}{|l|}{ Indwelling/external catheter } \\
\hline Yes & Reference & \\
\hline No & $0.86(0.59-1.24)$ & 0.411 \\
\hline Missing/unknown & $1.36(0.60-3.08)$ & 0.455 \\
\hline
\end{tabular}


Table 3 continued

\section{Characteristic}

Ostomy

Yes

No

Missing/unknown

Intermittent catheterization

Yes

No

Missing/unknown

None of the above bladder appliance

Yes

No

Missing/unknown

Urinary toileting program*

Yes

No

Missing/unknown

Response to toileting program*

No improvement

Decreased wetness

Completely dry (continent)

Missing/unknown

Current toileting program or trial*

Yes

No

Missing/unknown

Bowel continence*

Always continent

Occasionally incontinent

Frequently incontinent

Always incontinent

Not rated

Missing/unknown
Adjusted hazards ratio (95\% CI)

$P$ value

Reference

$1.46(1.01-2.12)$

0.044

$-$

Reference

$0.68(0.41-1.13)$

$-$

Reference

$0.96(0.68-1.34)$ 0.793

$-$

Reference

0.75 (0.58-0.97) 0.027

$0.76(0.66-0.86)$

$<0.0001$

Reference

$1.18(0.86-1.63)$

0.299

0.93 (0.60-1.43)

0.736

$1.02(0.83-1.26)$

Reference

0.95 (0.83-1.10)

0.510

$0.72(0.56-0.91)$

0.006

Reference

$1.00(0.92-1.09)$

0.988

1.09 (1.01-1.19)

0.036

$1.09(0.98-1.21)$

0.125

0.78 (0.57-1.08)

0.136

$1.32(0.59-2.94)$ 
Table 3 continued

\begin{tabular}{lll}
\hline Characteristic & Adjusted hazards ratio (95\% CI) & P value \\
\hline MDS cognition scale* & & \\
Intact & Reference & \\
Mild & $1.10(0.89-1.36)$ & 0.387 \\
Moderate & $1.04(0.90-1.20)$ & 0.575 \\
Moderate/severe & $1.02(0.80-1.30)$ & 0.867 \\
Severe & $\mathbf{1 . 2 5}(\mathbf{1 . 1 0 - 1 . 4 2 )}$ & $\mathbf{0 . 0 0 0 1}$ \\
Missing/unknown & $1.11(0.97-1.28)$ & 0.132 \\
Activities of daily living* & & \\
Independent & Reference & \\
Limited/extensive assistance & $1.14(0.99-1.32)$ & 0.061 \\
Dependent & $\mathbf{1 . 1 4}(\mathbf{1 . 0 5 - 1 . 2 3 )}$ & $\mathbf{0 . 0 0 3}$ \\
Missing/unknown & $1.00(0.81-1.25)$ & 0.965 \\
Depressed mood indicator & & \\
Yes & & \\
No & Reference & 0.964 \\
Missing/unknown & $1.00(0.86-1.17)$ & 0.010 \\
\hline
\end{tabular}

$A C B$ anticholinergic burden, $A I D S / H I V$ acquired immune deficiency syndrome/human immune deficiency virus, $L T N H$ long-term nursing home, $M D S$ minimum data set, $O A B$ overactive bladder, $C I$ confidence interval, $S D$ standard deviation All regression models were adjusted for predisposing, enabling and need factors

Significant values are bolded

*Based on MDS Admission Assessment and includes missing data

${ }^{* *}$ Based on enrollment data and includes missing data

$\dagger$ Categories of urinary incontinence were defined as: always continent (over a 7-day look-back period, resident was continent with urine, with no episodes of incontinence); occasionally incontinent (over a 7-day look-back period, resident was incontinent less than 7 episodes); frequently incontinent (over a 7 -day look-back period, resident was incontinent 7 or more episodes); always incontinent (over a 7-day look-back period, resident had no continent episodes); not rated (over a 7-day look-back period the resident had an indwelling catheter, condom catheter, ostomy or no urine output for the entire 7 days)

I Index antimuscarinic prescription refers to antimuscarinic medication that patients were newly started with upon nursing home admission

likely that the burden was much higher after antimuscarinic initiation and may have been a stronger predictor of discontinuation. Furthermore, patients with participation in a urinary toileting program, those who were dependent on ADL and those with frequent bowel incontinence were associated with higher discontinuation. As this is the first study that assessed antimuscarinic discontinuation among nursing home residents and adjusted for several need variables, comparison and contrast were not possible in the context of these variables.

Although this study provided insights regarding treatment patterns and predictors of antimuscarinic discontinuation, there are several limitations to be acknowledged. First, MDS 
measurements are assessed by nursing home providers and are used for resource use and reimbursement purposes rather than research. Therefore, there may be missing data if specific measures are not regarded as relevant, which may explain the high number of missing values for UI-related variables. Second, patients were assumed to have administered the medicationbased prescription fills prior to discontinuation. Hence, it should be acknowledged that time to discontinuation could have been much earlier than estimated here in the event that the medications were not taken. However, this would not differentially impact individual antimuscarinic agents. Third, as prescription medication use and discontinuation were based on Part D claims only during the nursing home stay, any medications provided by nursing homes as part of their care under the prospective payment system were not captured. The discontinuation was assessed based on Part D claims only; the issues with Part A coverage were not specifically addressed as Part A covers only the first 100 days and does not include prescription details; also, significant variability exists regarding the coverage source for Part D medications. However, this would not lead to differential bias in adherence/persistence. Fourth, as only antimuscarinics were examined in the present study, more research is needed to evaluate non-antimuscarinics such as mirabegron, sacral neuromodulation, posterior tibial nerve stimulation or the wider category of anticholinergics. As a result, the treatment and discontinuation patterns presented here can only be generalized to LTNH residents with OAB treated with antimuscarinics, and overall treatment rates may actually be higher than those estimated in the current study. Fifth, although the MDS may be a rich data source, several other factors not captured in the data source could not be included. Also, we decided to use a 3-month baseline period to increase the sample size. This could have led to some comorbidities not being captured. Other limitations include that the use of non-pharmacologic treatments (i.e., pads/briefs, or enemas/irrigation) was not captured, and as the data are limited to fee-forservice Medicare beneficiaries, the results cannot be generalized to uninsured nursing home residents or residents insured through Medicare Advantage plans. Lastly, although the ICD codes used here were consistent with those used to identify patients with $\mathrm{OAB}$ from other studies, there was the potential for misclassification, as evident by $5 \%$ of the study cohort identified with paralysis, which is typically associated with neurogenic detrusor overactivity rather than OAB. Furthermore, although males represented only one-fourth of $\mathrm{OAB}$ cases in the present study, it is possible that some of these cases were cases of BPH rather than OAB.

\section{CONCLUSION}

This retrospective cohort study found that $67 \%$ of LTNH residents with OAB discontinue antimuscarinic medications during nursing home stay, and several need characteristics were associated with discontinuation. Most importantly, the study found significant variation in medication discontinuation based on the index antimuscarinic agent. The study found that oxybutynin is most frequently used in nursing homes and discontinuation risk with oxybutynin is higher than for solifenacin and fesoterodine, but less than for toleterodine, trospium and darifenacin. Therefore, concerted efforts are needed to optimize and improve antimuscarinic medication use to improve quality of care of $\mathrm{OAB}$ in nursing homes.

\section{ACKNOWLEDGEMENTS}

Funding. This study, the Rapid Service and Open Access Fees were funded by Astellas Pharma Global Development, Inc.

Medical Writing and Editorial Assistance. We thank Meagan Harwood, MPH, for drafting, reviewing and editing this manuscript. Meagan Harwood is an employee of Broadstreet Health Economics \& Outcomes Research, which received payment from Astellas Pharma Global Development, Inc., for this assistance. 
Authorship. All named authors meet the International Committee of Medical Journal Editors (ICMJE) criteria for authorship for this article, take responsibility for the integrity of the work as a whole, and have given their approval for this version to be published.

Authorship Contributions. All authors contributed to the study design. Rajender R. Aparasu, Sneha Sura, and Jagadeshwara R. Earla performed the data analyses.

Disclosures. Aki Shiozawa, Daniel B. Ng and Carol R. Schermer are employees of Astellas Pharma Global Development, Inc. Rajender R. Aparasu, Jagadeswara R. Earla and Sneha Sura are employees of the University of Houston College of Pharmacy, who received funding from Astellas to conduct the study. The authors report no other disclosures regarding this work.

Compliance with Ethics Guidelines. The study protocol was approved by the University of Houston Committee for the Protection of Human Subjects. Informed consent was not required given the deidentified nature of the data and feasibility considerations for a large retrospective database such as Medicare.

Data Availability. Researchers may request access to anonymized participant-level data, trial-level data and protocols from Astellassponsored clinical trials at www.clinicalstudydatarequest.com. For the Astellas criteria on data sharing see: https://clinicalstudydatarequest. com/Study-Sponsors/Study-Sponsors-Astellas. aspx. The datasets generated during and/or analyzed during the current study are not publicly available as, per government regulations and the data use agreement, data from the Centers for Medicare and Medicaid Services cannot be shared.

Open Access. This article is licensed under a Creative Commons Attribution-NonCommercial 4.0 International License, which permits any non-commercial use, sharing, adaptation, distribution and reproduction in any medium or format, as long as you give appropriate credit to the original author(s) and the source, provide a link to the Creative Commons licence, and indicate if changes were made. The images or other third party material in this article are included in the article's Creative Commons licence, unless indicated otherwise in a credit line to the material. If material is not included in the article's Creative Commons licence and your intended use is not permitted by statutory regulation or exceeds the permitted use, you will need to obtain permission directly from the copyright holder. To view a copy of this licence, visit http:// creativecommons.org/licenses/by-nc/4.0/.

\section{REFERENCES}

1. Harris-Kojetin L, Sengupta M, Park-Lee E, et al. Long-term care providers and services users in the United States: data from the National Study of Long-term Care Providers, 2013-2014. National Center for Health Statistics. Vital Health Stat. 2016;3(38):1-12.

2. Moore KL, Boscardin WJ, Steinman MA, Schwartz JB. Patterns of chronic co-morbid medical conditions in older residents of US nursing homes: differences between the sexes and across the agespan. J Nutr Health Aging. 2014;18(4):429-36.

3. Tannenbaum C, DuBeau CE. Urinary incontinence in the nursing home: practical approach to evaluation and management. Clin Geriatr Med. 2004;20(3):437-52.

4. Wilson L, Brown JS, Shin GP, Luc KO, Subak LL. Annual direct cost of urinary incontinence. Obstet Gynecol. 2001;98(3):398-406.

5. Abrams P. Describing bladder storage function: overactive bladder syndrome and detrusor overactivity. Urology. 2003;62(5 Suppl 2):28-37.

6. Wein AJ, Rovner ES. Definition and epidemiology of overactive bladder. Urology. 2002;60(5 Suppl 1): 7-12.

7. Zarowitz BJ, Allen C, O'Shea T, Tangalos EG, Berner $\mathrm{T}$, Ouslander JG. Challenges in the pharmacological management of nursing home residents with overactive bladder or urinary incontinence. J Am Geriatr Soc. 2015;63(11):2298-307.

8. Hu TW, Wagner TH, Bentkover JD, Leblanc K, Zhou SZ, Hunt T. Costs of urinary incontinence and 
overactive bladder in the United States: a comparative study. Urology. 2004;63(3):461-5.

9. Chapple CR, Khullar V, Gabriel Z, Muston D, Bitoun CE, Weinstein D. The effects of antimuscarinic treatments in overactive bladder: an update of a systematic review and meta-analysis. Eur Urol. 2008;54(3):543-62.

10. Osterberg L, Blaschke T. Adherence to medication. N Engl J Med. 2005;353(5):487-97.

11. D'Souza AO, Smith MJ, Miller LA, Doyle J, Ariely R. Persistence, adherence, and switch rates among extended-release and immediate-release overactive bladder medications in a regional managed care plan. J Manag Care Pharm. 2008;14(3):291-301.

12. Pelletier EM, Vats V, Clemens JQ. Pharmacotherapy adherence and costs versus nonpharmacologic management in overactive bladder. Am J Manag Care. 2009;15(4 Suppl):S108-114.

13. Schabert VF, Bavendam T, Goldberg EL, Trocio JN, Brubaker L. Challenges for managing overactive bladder and guidance for patient support. Am J Manag Care. 2009;15(4 Suppl):S118-122.

14. Sexton CC, Notte SM, Maroulis C, et al. Persistence and adherence in the treatment of overactive bladder syndrome with anticholinergic therapy: a systematic review of the literature. Int J Clin Pract. 2011;65(5):567-85.

15. Rashid N, Vassilakis M, Lin KJ, Kristy R, Ng DB. Primary nonadherence to overactive bladder medications in an integrated managed care health care system. J Manag Care Spec Pharm. 2017;23(4): 484-93.

16. Vouri SM, Schootman M, Strope SA, Xian H, Olsen MA. Antimuscarinic use and discontinuation in an older adult population. Arch Gerontol Geriatr. 2019;80:1-11.

17. Research Data Assistance Center (ResDAC): Data Availability. 2018. https://www.resdac.org/cmsdata/file-availability. Accessed Aug 2018.

18. Centers for Medicare and Medicaid Services. Medicare standard analytical files: Identifiable data files. 2018. https://www.cms.hhs.gov/ IdentifiableDataFiles/02_StandardAnalyticalFiles. asp. Accessed Aug 2018.

19. Wei YJ, Simoni-Wastila L, Zuckerman IH, Brandt N, Lucas JA. Algorithm for identifying nursing home days using medicare claims and minimum data set assessment data. Med Care. 2014;54(11):e73-7.

20. Chancellor MB, Migliaccio-Walle K, Bramley TJ, Chaudhari SL, Corbell C, Globe D. Long-term patterns of use and treatment failure with anticholinergic agents for overactive bladder. Clin Ther. 2013;35(11):1744-51.

21. Scheife R, Takeda M. Central nervous system safety of anticholinergic drugs for the treatment of overactive bladder in the elderly. Clin Ther. 2005;27(2): 144-53.

22. AHFS Drug Information Essentials. Bethesda, MD: American Society of Health-System Pharmacists, 2018.

23. Sussman D, Yehoshua A, Kowalski J, et al. Adherence and persistence of mirabegron and anticholinergic therapies in patients with overactive bladder: a real-world claims data analysis. Int J Clin Pract. 2017;71(3-4):e12824.

24. Wagg A, Franks B, Ramos B, Berner T. Persistence and adherence with the new beta-3 receptor agonist, mirabegron, versus antimuscarinics in overactive bladder: early experience in Canada. Can Urol Assoc J. 2015;9(9-10):343-50.

25. Peterson AM, Nau DP, Cramer JA, Benner J, Gwadry-Sridhar F, Nichol M. A checklist for medication compliance and persistence studies using retrospective databases. Value Health. 2007;10(1):3-12.

26. Andersen RM. Revisiting the behavioral model and access to medical care: does it matter? J Health Soc Behav. 1995;36(1):1-10.

27. Andersen R, Newman JF. Societal and individual determinants of medical care utilization in the United States. Milbank Mem Fund Q Health Soc. 1973;51(1):95-124.

28. Chapple CR, Nazir J, Hakimi Z, et al. Persistence and adherence with mirabegron versus antimuscarinic agents in patients with overactive bladder: a retrospective observational study in UK clinical practice. Eur Urol. 2017;72(3):389-99.

29. Elixhauser A, Steiner C, Harris DR, Coffey RM. Comorbidity measures for use with administrative data. Med Care. 1998;36(1):8-27.

30. Campbell N, Boustani M, Limbil T, et al. The cognitive impact of anticholinergics: a clinical review. Clin Interv Aging. 2009;4:225-33.

31. Offermans MP, Du Moulin MF, Hamers JP, Dassen T, Halfens RJ. Prevalence of urinary incontinence and associated risk factors in nursing home residents: a systematic review. Neurourol Urodyn. 2009;28(4):288-94.

32. Morris JN, Fries BE, Mehr DR, et al. MDS cognitive performance scale. J Gerontol. 1994;49(4): M174-182. 
33. Morris JN, Fries BE, Morris SA. Scaling ADLs within the MDS. J Gerontol A Biol Sci Med Sci. 1999;54(11):M546-553.

34. American Geriatrics Society Beers Criteria Update Expert P. American Geriatrics Society 2019 updated AGS beers criteria(R) for potentially inappropriate medication use in older adults. J Am Geriatr Soc. 2019;67(4):674-94.

35. Odeyemi IA, Dakin HA, O'Donnell RA, Warner J, Jacobs A, Dasgupta P. Epidemiology, prescribing patterns and resource use associated with overactive bladder in UK primary care. Int J Clin Pract. 2006;60(8):949-58.

36. Altman D, Granath F, Mattiasson A, Falconer C. Anticholinergic drug use for overactive bladder in Sweden: a nationwide pharmacoepidemiological study. Int Urogynecol J Pelvic Floor Dysfunct. 2009;20(11):1285-91.

37. Ju R, Garrett J, Wu JM. Anticholinergic medication use for female overactive bladder in the ambulatory setting in the United States. Int Urogynecol J. 2014;25(4):479-84.

38. Parsons C, Lapane K, Kerse N, Hughes C. Prescribing for older people in nursing homes: a review of the key issues. Int J Older People Nurs. 2011;6(1): 45-544.

39. Kachru N, Carnahan RM, Johnson ML, Aparasu RR. Potentially inappropriate anticholinergic medication use in older adults with dementia. J Am Pharm Assoc (2003). 2015;55(6):603-12.

40. Pazan F, Gercke Y, Weiss C, Wehling M, Raters F. The US-FORTA (Fit fOR The Aged) list: consensus validation of a clinical tool to improve drug therapy in older adults. J Am Med Dir Assoc. 2020;21(3): e439.

41. Gray SL, Anderson ML, Dublin S, et al. Cumulative use of strong anticholinergics and incident dementia: a prospective cohort study. JAMA Intern Med. 2015;175(3):401-7.

42. Richardson K, Fox C, Maidment I, et al. Anticholinergic drugs and risk of dementia: case-control study. BMJ. 2018;361:k1315.

43. Tannenbaum C, Paquette A, Hilmer S, Holroyd-Leduc J, Carnahan R. A systematic review of amnestic and non-amnestic mild cognitive impairment induced by anticholinergic, antihistamine, GABAergic and opioid drugs. Drugs Aging. 2012;29(8):639-58.

44. Kay G, Crook T, Rekeda L, et al. Differential effects of the antimuscarinic agents darifenacin and oxybutynin ER on memory in older subjects. Eur Urol. 2006;50(2):317-26.

45. Abrams P, Kelleher CJ, Kerr LA, Rogers RG. Overactive bladder significantly affects quality of life. Am J Manag Care. 2000;6(11 Suppl):S580-590. 